Gérald Quatrehomme, ${ }^{1}$ M.D.; Stéphane Cotin, ${ }^{2}$ B.S.; Gérard Subsol, ${ }^{2}$ Ph.D.;

Hervé Delingette, ${ }^{2}$ Ph.D.; Yves Garidel, ${ }^{3}$; Gilles Grévin, ${ }^{4}$ B.S.; Martha Fidrich, ${ }^{2}$ B.S.; Paul Bailet, ${ }^{4}$;

and Amédée Ollier, ${ }^{4}$ M.D.

\title{
A Fully Three-Dimensional Method for Facial Reconstruction Based on Deformable Models
}

REFERENCE: Quatrehomme G, Cotin S, Subsol G, Delingette H, Garidel Y, Grévin G, Fidrich M, Bailet P, Ollier A. A fully threedimensional method for facial reconstruction based on deformable models. J Forensic Sci 1997;42(4):649-652.

ABSTRACT: Two facial models corresponding to two deceased subjects have been manually created and the two corresponding skulls have been dissected and skeletonized. These pairs of skull/facial data have been scanned with a CT scanner, and the computed geometric three-dimensional models of both skulls and facial tissue have been built. One set of skull/facial data will be used as a reference set whereas the second set is used as ground truth for validating our method. After a semi-automatic face-skull registration, we apply an original computing global parametric transformation $\mathrm{T}$ that turns the reference skull into the skull to be reconstructed. This algorithm is based upon salient lines of the skull called crest lines: more precisely the crest lines of the first skull are matched to the crest lines of the second skull by an iterative closest point algorithm. Then we apply this algorithm to the reference face to obtain the "unknown" face to be reconstructed. The reliability and difficulties of this original technique are then discussed.

KEYWORDS: forensic science, forensic anthropology, human identification, facial reconstruction, computer imaging, casting, forensic pathology

Facial reconstruction bas emerged as an increasingly important tool in forensic pathology and anthropology, generating a lead for positive identification from any classical comparative technique. In this field, computers have been mostly used to store and retrieve images from facial databases. More advanced softwares propose to modify the presence of facial hair or facial features of a given reference facial image. Those softwares do not treat the problem of reconstruction from a skull and furthermore are essentially two-dimensional.

The aim of this work is to experiment a new original method that extends the use of computers to the problem of facial reconstruction: given a skull of a cadaver, we intend to reconstruct by computer a three-dimensional facial model of the deceased person. There are actually several issues associated with this goal. The first issue

${ }^{1}$ Forensic pathologist, Maître de Conférences des Universités, Laboratory of Forensic Medicine, Faculté de Médecine, Nice Cédex 2, France.

${ }^{2}$ Epidaure Group, National Institute for Research for Informatics and Automatics, Sophia-Antipolis, France.

${ }^{3}$ Laboratory of Ethnology, Faculté des Lettres, Nice Cédex 2, France.

${ }^{4}$ Laboratory of Physical Anthropology, National Center of Scientific Research, Draguignan, France.

Received 15 July 1996; and in revised form 14 Oct. 1996; accepted 11 Nov. 1996. consists in acquiring, processing, and visualizing the three-dimensional models of skulls and faces. The second issue concerns the actual facial reconstruction task, i.e., proposing a facial model of a cadaver based on the shape of its skull. In the near future, we plan to include a third issue consisting in editing the characteristics of the facial appearance such as the hair and facial features.

The benefit of a computerized facial reconstruction would be threefold. First, it would surely ease the procedure of the reconstruction itself. We probably cannot conceive to make this procedure fully automatic; however, it is clear that computer software can greatly reduce the amount of time spent by the forensic scientist for proposing a facial model. The second benefit consists in producing several possible facial models from a given skull depending on varying parameters, particularly with the hypothesis that the person was fat, slim, old, or young. Finally, the computer methodology we are proposing will produce a three-dimensional model of the face, and the ability to observe a person under several angles should increase the probability of identification of an individual.

\section{Methods}

The general goal that we want to achieve is to have a model-based reconstruction of the face of a given skull. A database of head models (i.e., both skulls and faces) with their personal characteristics (age, sex, race, and nutrition status) is required. We would like to choose from the database the most "similar" head as a reference in such a way that the given and the reference skulls should have roughly the same characteristics. Under the assumption that "if skulls have similar forms, the corresponding faces should have main characteristics in common," we propose a face of the given skull whose reconstruction is guided by the reference head. Presently, only a simplified version of this general scheme is implemented. We are given two sets of models: the first set $\mathrm{E} 1=\{\mathrm{S} 1, \mathrm{~F} 1\}$, consisting of a skull model S1 and a facial model F1, is to be considered as the head with unknown face, whereas the second set E2 $=\{\mathrm{S} 2, \mathrm{~F} 2\}$ is the reference head with a known skull S2 and facial F2 models. In order to evaluate our method, we have to build a facial model $\mathrm{F}^{*}$ given the skull $\mathrm{S} 1$. The success of reconstruction can be gained from the comparison of the proposed facial model $\mathrm{F}^{*}$ with the real shape of the face F1. Because the reference set guides the reconstruction algorithm, E2 should ideally present most characteristics of .'normality" and roughly belong to the same age, sex, race, and nutrition status as the skull S1. 


\section{Data Acquisition}

The digitalization of the two datasets has been performed in the Department of Radiology of Cannes, France. Using CT scanning of a living subject is impossible, because we need very thin CT scan slices, and the amount of radiations received by the subject would be important. CT scanning of the head of a cadaver (i.e., the skull and face together) has some problems, especially in regard to ethical issues, as well as practical and important technical issues (artifacts from metallic material in teeth). Therefore, we have chosen to digitalize the skull and a mold of the face separately. Forensic casting techniques have been developed in the Laboratory of Forensic Medicine of Nice, in collaboration with the Laboratory of Ethnology of Nice, France $(1,2)$. CT scanning of a casting of the face is easy, though some problems persist. The first one is the necessity of rebuilding the posterior part of the casting of the head, because our casting technique deals only with the face: This problem had been previously overcome through a classical clay reconstruction. The second one is the opening of the mandibule which must be always in the same angle, both on the cadaver as well as on the skeletonized skull, and we have to find devices to overcome this task.

The CT-scan produces a set of slices with an interstice distance that may reach $1 \mathrm{~mm}$. Those slices are stacked into volumetric images, then a skull and facial model are produced by using an isosurface extraction algorithm (3). The Epidaure group in Sophia- Antipolis, France, deals with three-dimensional image processing and was able to obtain four distinct models corresponding to two skulls S1, and S2 (S2: reference skull) and two faces F1, and F2 (F2: reference face). However, when we acquire the shape of the skull and the face in a separate process, it is necessary to register those two models with each other. We accomplish the face-skull registration in a semi-automatic manner. It is extremely important that the registration is performed precisely because succeeding computation will rely on the relative distances between the skull and the face. Furthermore, we have used a visualization tool called AVS (AVS-UNIRAS, France) and developed a particular interface dedicated to the manipulation of the skull and facial models.

\section{Methods of Reconstruction}

The first method consists in acquiring some landmarks on the skull $\mathrm{S} 1$ to be reconstructed. The surface win then deform in order to fit as closely as possible the estimated facial landmarks while keeping as much its original shape as possible. Many cranial and facial landmarks are certainly needed to drive the deformation. The second method is based on a global parametric algorithm $\mathrm{T}$ that transforms the reference skull S2 into the skull S1 :

$$
T(S 2)=S 1
$$

and we make the following hypothesis: the application of the transformation on the reference face P2 should give a facial model that is close to the real face F1:

$$
T(F 2) \approx F 1
$$

The application of the global transformation $\mathrm{T}$ may be seen as warping one facial model onto the other. In our method, the computation of the transformation $\mathrm{T}$ is not based on the whole set of points describing the skull; it is based only on some salient lines called crest lines $(4,5)$. Mathematically, those lines are defined by differential invariants and correspond to lines of absolute maxima of the largest principal curvature. On a skull, the crest lines correspond to, for example, the outlines of the mandibule, the orbits, the cheekbones, or the temples (Fig. 1), and also, inside the cranium, the sphenoid and temporal bones as well as the foramen magnum. Given two sets of crest lines extracted from two different skulls, we want to find the transformation $\mathrm{T}$ so that the crest lines of the first skull are matched to the crest lines of the second skull.

The sets of lines are very different in orientation, size, number shape, topology, and discretization, which requires a sophisticated registration algorithm: the process consists of iteratively applying transformations, based on a local point matching, to the set of crest lines S2 to superimpose it on the set $\mathrm{S} 1$. We use first rigid transformations to align the two sets, then affine transformations to retrieve the scales, quadratic transformations to model some natural deformations (6) or 3D spline transformations (7) which allow local deformations. At each iteration, the points of the lines of S2 are linked with their closest neighbor in $\mathrm{S} 1$ according to the Euclidean distance, which gives a list of couples of registered points. At the end, we obtain a transformation $\mathrm{T}$ that can be applied on the whole set of points of the skull, i.e., not only on the crest lines. Furthermore, unlike the former method, this one does not rely upon any craniofacial distances but merely on the homology between the two datasets. Details can be found in Subsol et al. (8) with an application to atlas building.

\section{Results}

So far, we were able to make enough experiments only based on the second method. We present preliminary results from two sets consisting of two facial molds and two dry skulls. Face to skull registration was performed, but problems occurred due to different degrees of opening of the mandibule. Furthermore, the two faces had a very different morphology. Therefore the hypothesis of close similarity of age and main characteristics of the face was not verified. Figure 2 shows the comparison between the face directly reconstructed from the skull by the computer algorithm (left), and the actual face (right), or more precisely the mold of the actual face of the

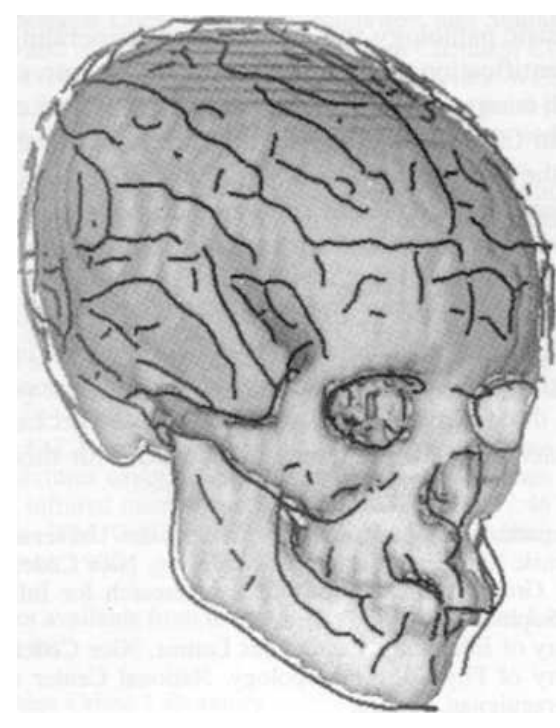

FIG. l-External crest lines on the skull. 

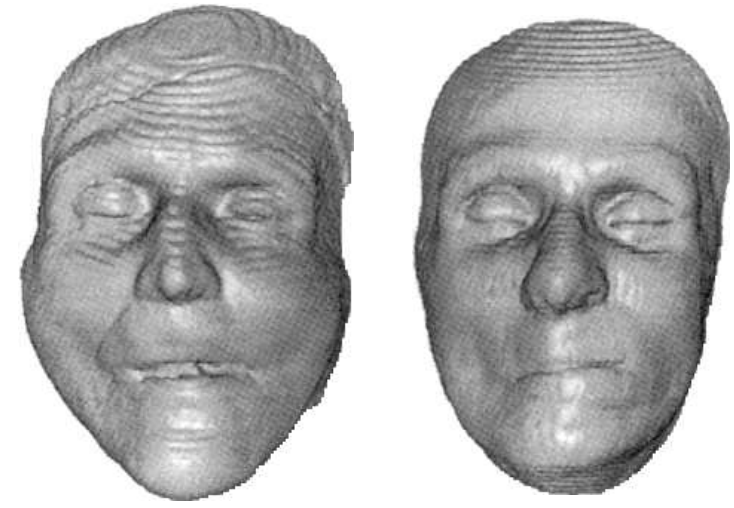

FIG. 2-Comparison between 3D reconstructed face (left), and actual mold of the face (right).

deceased. We can observe that the upper part of faces correspond fairly well, however some differences can be perceived near the chin mainly due to the above mentioned problems.

\section{Discussion}

Forensic facial reconstruction is a technique of approximating the facial appearance of an individual in life for purposes of further individual identification. Various methods have been described, from two-dimensional to three-dimensional, non-computerized to computerized methods. Review of most of these methods have been presented in textbooks of forensic anthropology $(9,10)$, and a survey bas been performed by Aulsebrook et al. in 1995 (11). Reconstruction techniques were first developed by German anatomists in the late 1800 s in order to identify historical personages. Later the Russian anthropologist Gerasimov $(12,13)$ developed the clay threedimensional method and used it in forensic cases. Since the landmark work of Snow in 1970 (14), the field bas rapidly grown worldwide $(15,16)$. Evaluation of the reliability of the clay dimensional method is rarely reported on. The first attempt at a scientific testing was by Snow et al. in 1970 (14). We have then to quote Aulsebrook and Van Rensburg (17), Ubelaker (18), Krogman (19), Neave (20), and Quatrehomme (21). The most extensive work was of Helmer et al. $(22,23)$ that conducted a double-blind trials in which two independent examiners reconstructed soft tissues on casts of 12 skulls. In fact results are varying, from poor to excellent, and these variations have been previously analyzed $(11,24)$. Nevertheless two-dimensional methods as sketching the facial features (25), or performing lateral craniographic method (26) are also currently used, as well as superimposition techniques.

Most of the methods are based on the knowledge of mean values of facial soft tissue thickness measured at selected anatomical landmarks, as well as knowledge of particular morphologic features. In fact facial reconstruction is not easy, because there are many facial variations, particularly according to the nutritional status of the individual and the different rates and intensities of ageing. Furthermore, the details of the nose, eye, ear, lips, and chin probably cannot be constructed exactly from the skull characteristics. The current tendency in the forensic facial reconstruction may be the development of new computerized methodologies, with the advantages of swiftness and reduction of subjectivity. The possibility and validity of computer-aided facial reconstruction have been investigated by different authors. Perper (27) developed a facial imaging reconstructive morphography system that permitted the construction of facial features based on precise cephalometric measurements of X-ray of the skull. Ubelaker (28) reported the interest of computer-assisted facial reconstruction by using the kit that had been developed for ageing missing individuals. Vanezis (29) applied a three-dimensional computer graphics technique for facial reconstruction and achieved comparison with sculpting techniques. Miyasaka (30) developed a computer-aided facial reconstruction using the same methodological way as the two- dimensional drawing method, concluding that the resulting image was a great improvement on images made by the conventional clay and drawing method, both in the operational aspect and in the flexibility of creating multiple versions. Lambrecht (31) utilized a software permitting separate visualization of soft tissues, bones, and air spaces through computed tomographic data and video visualization. Evenhouse (32) developed a 3D computer-aided method, by drafting the soft tissue profile as following the procedures suggested by Georges (26), and connecting the end-points of the tissue depth markers, what permitted to generate a series of polygons to comprise a rudimentary soft tissue surface or face form.

Our computerized method is original because it is not based on altering the skull by using specific landmarks and average facial tissue thickness. It is based on: (i) an original global parametric algorithm, which involves crest lines, corresponding mathematically to lines of absolute maxima of curvature; (ii) this algorithm involves and is calculated from the transformation of the reference skull into the skull to be reconstructed; then the same algorithm is applied for the transformation of the reference face into the face to be reconstructed. It runs from skull to skull, and then from face to face. This is obviously an unusual way, because usually the computerized methods run from skull to face. By this method we were able to obtain a facial reconstruction which is quite close to the real face. The interest of this method is its swiftness, and the fact that the computer directly generates a three-dimensional facial model, contrary to the different methods described in the international literature. We are able to translate, rotate, zoom, or alter this 3D-image in different ways, and the possibility of matching a perspective in a photograph of a missing person is very useful. We are also able to interactively select with a good precision a set of landmarks on the skull, and if necessary to compute the projection of these landmarks along the normal direction on the face. We can easily compute the distance between face and landmarks and, for instance, compare those distances with existing reference tables.

Current results are promising and we believe that it could be greatly improved by the use of larger datasets covering several categories of individuals. Nevertheless, some problems have to be dealt with such as: accurate registration of skull and facial model, accurate and invariable position of the mandibule, and the method needs further investigations and improvements. We hope that this original technique will make a contribution to the field of forensic identification.

\section{References}

1. Quatrehomme G, Garidel Y, Grevin G, Liao ZG, Bailet P, 01lier A. Method for identifying putrefied corpses by facial casting. Forensic Sci Int 1995;74(1-2):115-24. 
2. Quatrehomme G., Garidel Y, Grevin G, Liao ZG, Boublenza A, Ollier A. Facial casting as a method to help identify severely disfigured corpses. J Forensic Sci 1996;41(3):324--6.

3. Thirion JPh, Gourdon A. The 3D marching lines algorithm: New results and proofs. Technical Report no.1881, 1993;Submitted to CGVIP.

4. Monga O, Benayoun S, Faugeras OD. Using partial derivatives of 3D images to extract typical features. In CVPR 1992.

5. Thirion JPh, Gourdon A. Computing the differential characteristics of isointensity surfaces. CVGIP 1995;190-202.

6. Greitz T, Bohm CH, Holte S, Eriksonn. A computerized brain atlas: construction, anatomical content and some applications. J Comput Assisted Tomog 1991;15(1):26-38.

7. Szeliski R, Lavallee S. Matching 3D anatomical surfaces with non- rigid deformations using Octree-Splines. In: IEEE, workshop on biomedical image analysis. Seattle (Washington), 1994;144-53.

8. Subsol G, Thirion JPH, Ayache N. A general scheme for automatically building 3D morphometric anatomical atlases: Application to a skull atlas, medical robotics and computer assisted surgery. Baltimore, Maryland (U.S.A.), 1995.

9. Krogman WM, Iscan MY. The human skeleton in forensic medicine. 2nd ed. Springfield, IL: Charles C Thomas, 1986.

10. Stewart TD. Reconstruction of facial soft parts. In: Stewart TD. Essentials of forensic anthropology. Springfield, IL: Charles C Thomas, 1979;25574.

II. Aulsebrook WA, Iscan MY. Superimposition and reconstruction in forensic facial identification: A survey. Forensic Sci Int 1995;75(2-3): 101-20.

12. Gerasimov MM. The face finder. J.B. Lippincott Co. Philadelphia, PA, 1971.

13. Gerasimov MM. Principles of reconstruction of the face on the skull, 1949, Moscow, Nauka (in Russian), cited by Lebedinskaya GV, Balueva TS, Veselovskaya EV. Principles of facial reconstruction. In: Iscan MY, Helmer RP, editors. Forensic analysis of the skull. New-York:WileyLiss, 1986;183-98.

14. Snow CC, Gatliff BP, McWilliams KR. Reconstruction of facial features from the skull: An evaluation of its usefulness in forensic anthropology. Am J Physic AnthropoI1970;33:221-8.

15. Gatliff BP, Snow CC. From skull to visage. J Biocommun 1979;6(2):2730.

16. Gatliff BP. Facial sculpture on the skull for identification. Am J Forensic Med Pathol 1984;5(4):327-32.

17. Aulsberook WA, Van Rensburg JHJ. An evaluation of two techniques used for facial reconstruction in forensic anthropology. Abstract of lecture delivered at the 16th Annual Congress of the Anatomical Society of South Africa. S Afr J Sci 1986;82:448.

18. Ubelaker DH. Human skeletal remains. Excavation, analysis, interpretation. Manuals of Archaeology, 2nd ed. Taraxacum Washington $1989 ; 124$.

19. Krogman WM. Role of the physical anthropologist in the identification of human skeletal remains, Part I. FBI Law Enforcement Bull 1943;12:1228.
20. Neave RAH. Facial reconstruction: A study of its accuracy and reproducibility. Am I Phys Anthropol 986;69:244 (abstract).

21. Quatrehomme G, Garidel Y, Bailet P, Liao ZG, Grevin G, Ollier A. An attempt of scientific evaluation in facial reconstruction: Preliminary results. Proceedings of the XVIth Congress of the International Academy of Legal Medicine and Social Medicine. 31 May-2 June 1994. Strasbourg, France,

22. Helmer RP, Rohricht S, Petersen D, Mohr F. Plastic face reconstruction as a possibility for identifying skulls (1): An evaluation of the reliability of the reconstruction technique by a double-blind trial. Arch Kriminol 1989;184:75-83.

23. Helmer RP, Rohricht S, Petersen D, Mohr F. Assessment of the reliability of facial reconstruction. In: Iscan MY, Helmer RP, editors. Forensic analysis of the skull. New- York:Wiley-Liss, 1986; 229-46.

24. Caldwell PC. New questions (and some answers) on the facial reproduction techniques. In: Reichs KI, editor. Forensic osteology: Advances in the identification of human remains. Springfield, IL: Charles C Thomas, 1986;229-55.

25. Taylor KT. Technique of facial reconstruction drawing. Proceedings of International Symposium on the Forensic Aspects of Mass Disasters and Crime Scene Reconstruction. June 23-29, 1990. FBI Academy, Quantico, Virginia.

26. George RM. The lateral craniographic method of facial reconstruction. J Forensic Sci 1987;32(5):1305-30.

27. Perper JA, Patterson GT, Backner JS. Face imaging reconstructive morphography. Am I Forensic Med Pathol1988;9:126-38.

28. Ubelaker DH, O'Donnell G. Computer-assisted facial reproduction. J Forensic Sci 1992;37(1):155-62.

29. Vanezis P, Blowes RW, Linney AD, Tan AC, Richards R, Neave R. Application of 3-D computer graphics for facial reconstruction and comparison with sculpting techniques. Forensic Sci Int 1989;42:69-84.

30. Miyasaka S, Yoshino M, Imaizumi K, Seta S. The computer-aided facial reconstruction system. Forensic Sci Int 1995;74:155-65.

31. Larnbrecht JT, Brix F, Gremmel H. Three-dimensional skull identification via computed tomographic data and video visualization. In: Iscan MY, Helmer RP, editors. Forensic analysis of the skull. New York:WileyLiss, 1986;97-104.

32. Evenhouse R, Rasmussen M, Sadler L. Computer-aided forensic facial reconstruction. J Biocommunication 1992;19(2):22-8.

Additional information and reprint requests:

Quatrehomme Gérald, M.D.

Forensic Pathologist, Maître de Conférences des Universités

Laboratory of Forensic Medicine

Faculté de Médecine

Avenue de Vallombrose, 06107 Nice Cédex 2

France 